\title{
Rapid evolution by spontaneous mutation increases genetic diversity facilitating plant
} population survival

3 Henning Nottebrock ${ }^{1}$, Mao-Lun Weng ${ }^{1}$, Matthew T. Rutter ${ }^{2}$ \& Charles B. Fenster ${ }^{1}$

$5{ }^{1}$ Department of Biology and Microbiology, South Dakota State University, Brookings, SD USA

${ }^{2}$ Department of Biology, College of Charleston, Charleston, SC USA

Abstract

Using a mechanistic eco-evolutionary trait-based neighborhood-model, we quantify the impact of mutations on spatial interactions to better understand the potential effect of niche evolution

11 through mutations on the population dynamics of Arabidopsis thaliana. We use 100 twenty-

12 fifth generation mutation accumulation (MA) lines (genotypes) derived from one founder genotype to study mutational effects on neighbor responses in a field experiment. We created

14 individual-based maps (15,000 individuals), including phenotypic variation, to quantify mutational effects within genotypes versus between genotypes on reproduction and survival. At small-scale, survival is enhanced but reproduction is decreased when a genotype is

17 surrounded by different genotypes. At large-scale, seed set is facilitated by different genotypes while the same genotype has either no effect or negative effects. Mutations may provide a mechanism for plants to quickly evolve niches and may drive competition, facilitation and selection with profound consequences for future population and community dynamics. 
Introduction

22

23

24

25

26

27 2016).

A complete understanding of population and community dynamics requires linking intraspecific genetic diversity with spatial ecological interactions (Bolnick et al. 2011, Genung et al. 2011). Evolutionary mechanisms -- mutation, drift, gene flow and selection -- are responsible for intraspecific genetic variation contributing to both ecological structure and species diversity (Hart et al. 2016). These community properties impact ecological mechanisms such as competition and facilitation (Whitlock 2014). Competition and facilitation in turn affect population and community dynamics that depend on variation in demographic rates (Chase et al 2002, Solivers et al 2015). Demographic rates are influenced by spatial interactions of genetic variation and this variation may contribute to species coexistence: genotypes can hinder or favor the survival of each other by using similar or different resources with negative or positive consequences for coexisting species (Hart et al. 2016, Hausch et al. 2018). Intraspecific genetic variation contributing to individual trait variation and environmental adaptability may promote species coexistence by both increasing habitat heterogeneity and altering competitive hierarchies (Violle et al. 2012, Ehlers et al. 2016). However, intraspecific genetic variation may hinder species coexistence when intraspecific genetic variation is diminished by competition between individuals (Hart et al 2016). To fully understand the causes of coexistence mechanisms, we need to integrate intraspecific evolutionary mechanisms with intraspecific ecological mechanisms underlying spatial species interactions (Bolnick et al. 2011, Ehlers et al. 
The number of studies exploring eco-evolutionary dynamics and their potential

43

44

feedbacks on population and community dynamics has dramatically increased over the last two decades (Shefferson and Salguero-Gomez 2015). An important current issue in community and ecosystem genetics research is determining the relevance of intraspecific genetic variation and genetic differentiation (divergence) to ecological and evolutionary processes at the community and ecosystem level (Genung et al. 2011, Pujol et al. 2018). Quantifying intraspecific trait variation defining the fundamental niches of species is an important link between ecology and evolution (Violle and Jiang 2009). Spatial structure is another important component of realistic eco-evolutionary dynamics, such that in spatially structured populations, selection is determined by the interplay between demographic and genetic structures (Lion et al. 2011). Demographic structure describes the spatial distribution of individuals through birth, death and migration resulting in spatial patterns, while genetic structure describes the spatial distribution of genotypes. The amount and spatial pattern of genetic variation may constrain evolution of traits influencing competitive ability of individuals (Wilson 2014). Moreover, competitive abilities depend on species' niches. The strength of competition is determined by how much individual niches overlap with each other (Hutchinson 1957, Holt 2009) and how long coexistence has occurred (Connell 1980). interspecific genetic and spatial interactions driven by environmental conditions. Thus, intraspecific trait variability shaped by intraspecific genetic variation can influence ecological mechanisms driving variation among individual persistence (Lankau 2009). Only when the 
spatial relationship between traits and demographic parameters is explicitly described can robust hypotheses about the effects of individual variation on competitive outcomes be accurately formulated (Hart et al. 2016). Especially in controlled common garden environments, genetic variation in one species can have predictable and heritable effects on associated communities and ecosystems (Carr and Dudash 1995; Whitham et al. 2003, 2006; Johnson \& Stinchcombe 2007; Bailey et al. 2009; Johnson, Vellend \& Stinchcombe 2009). Hence, coupling evolutionary genetics with community ecology may advance our understanding of species interactions and population and community dynamics (Baron et al. 2016), especially for populations and communities that suffer environmental change (Bellard et al. 2012).

(1)

An important gap in our understanding of the link between evolutionary and ecological processes is the nearly complete absence of data quantifying how rapidly genetic variation governing within species competitive hierarchies evolves (Hausch et al. 2018). An important source of novel population genetic variation is mutation. Therefore, we aim to investigate the link between evolutionary genetics and spatial ecological interactions of mutation accumulation lines (MA lines) in the model plant organism Arabidopsis thaliana (Brassicaceae) to advance our understanding of the genetic origins of population and community dynamics, contributing to a fuller understanding of the maintenance of biodiversity. We take advantage of 25th generation A. thaliana mutation accumulation (MA) lines that were planted under field conditions in years 2004 and 2005 with spatial records of each individual each year (e.g., Rutter et al. 2010; 2012, Rutter et al. 2018) (Fig. 1). 
87 MA line (within genotypes) or different MA lines (between genotypes) to quantify the evolution

88 of intraspecific genetic variation through the accumulation of spontaneous mutations. We

89 implement trait-based neighborhood models (Nottebrock et al. 2017a, Nottebrock et al. 2017b,

90 Lachmuth et al. 2017) to understand ecological mechanisms of within and between MA lines

91 genetic variation to examine eco-evolutionary dynamics (Lion 2018). Specifically, we ask: 1) Do

92 spontaneous mutations rapidly introduce enough genetic variation among MA lines to influence

93 competition and facilitation of $A$. thaliana? 2) Do spontaneous mutations of MA lines

94 contribute to competition, facilitation and selection promoting differential MA line survival and

95 reproduction at different spatial scales? 3) How may competitive and facilitative effects

96 potentially determine population and community dynamics?

97 
Methods

MA lines are generated from a single nearly homozygous individual founder and cultivated via limited effective population number. In the case of $A$. thaliana, this occurs through single seed descent, resulting in $\mathrm{Ne}=1$. Thus typical $\mathrm{MA}$ line cultivation results in an unbiased sample of mutation effects ranging from deleterious to advantageous, although lethal mutations are excluded (Lynch and Walsh 1998). Each MA line accumulates independent spontaneous mutations. After the propagation of a set of MA lines through multiple generations, the genetic differences among the MA lines and between those lines and the founder reflect the input of mutation. Significant MA line effects for multiple traits, including performance and trait measures, were found under both field and greenhouse conditions (Rutter et al. 2010; Roles et al. 2016, Rutter et al. 2018). Each of the MA lines in our experiment is fixed for an average of 20 different sequence level mutations, single nucleotide mutations (SNMs) and indels combined (Ossowski et al 2010, Rutter et al. 2012, Weng et al. 2019).

\section{MA lines and field experiments}

We used survival and seed set data of $A$. thaliana MA lines and the founder as assessed in field experiments in 2004 and 2005 from Rutter et al. (2010, 2012 and 2018) planted in a randomized design (Fig. 1). Rutter et al. (2010, 2012) planted seedlings of 100 MA lines and the founder at the four-leaf stage, approximately two weeks post germination, into a secondary successional field at Blandy Experimental Farm (BEF) in Virginia $\left(39^{\circ} \mathrm{N}, 78^{\circ} \mathrm{W}\right)$. Each of the 100 MA lines was used to found up to five sublines to minimize biases due to maternal effects 
introduced by the specific location within the greenhouse. We founded six sublines from each of the six lines representing the premutation founder genotype. In 2003, subline plants were used to generate all seed utilized in all field experiments. In each planting, 7504 individuals were planted, 7000 individuals of 100 MA lines (70 replicates per MA line, 14 replicates per subline) and 504 individuals of the founder (14 replicates per subline). The planting environment corresponds to a spring ephemeral life-history, where plants germinate and complete the life-cycle in the spring. At the time of planting, vegetation was scant but present. By harvest, the $A$. thaliana individuals were dwarfed by naturally occurring vegetation. The plot was arranged in 14 spatial blocks with each containing 12 subblocks (Fig. 1B) (total plot area approximately $35 \times 25 \mathrm{~m}$ ). Each block included one seedling from each subline and in total 7504 individuals. We used the spatial information of each individual within the described design and created a raster of all plant individuals with R packages (raster, maps, maptools). We used individual-based maps neighborhood matrices with exact spatial and trait information of each genotype. If all five sublines did not produce enough seedlings to distribute in all blocks, seedlings from other sublines of the same MA line were overrepresented in blocks to maintain the same overall number of plants per MA line. Plants dying within the first 3 days of transplant (about 50 plants) were considered to have died from transplant shock and were replaced with another plant from the same MA line. Plants were censused weekly for survival. Plants were harvested by late May, by which time they had senesced. In 2004 a total of 5915 individuals including 394 founders survived. In 2005, a total of 4506 individuals survived 
140 including 302 founders. Plants were oven dried and biomass was measured. All fruits produced

141 by each plant were counted and in combination with seed production used as the

142 measurements of seed set. For our analysis, we used measurements of two response variables

143 representing an important part of the life history of $A$. thaliana to calculate direct

144 neighborhood effects on: a) the survival rate of plant individuals and b) individual seed set from

145 plants that survived and produced fruits. 
Statistical Analyses

148

149

150

We use eco-evolutionary trait-based neighborhood models that include intraspecific genetic variation and phenotypic variation expressed by plant trait biomass to analyze competition and facilitation between individuals of $A$. thaliana. We analyzed plant survival rate and focal seed set measurements from years 2004 and 2005 separately. For each year, we considered all individuals as focal plants in the analysis based on individual-based neighborhood matrices to analyze 1) rate of plant survival to reproduction and 2) seed set of those plants that survived to reproduction. We analyzed neighboring plants to focal plants in a radius of $80 \mathrm{~cm}$ (small-scale) or $200 \mathrm{~cm}$ (large-scale) of a given focal plant as two spatial scales in the neighborhood analyses (Fig. 1) to quantify selection, competition or facilitation between plants depending on their genotypic and phenotypic variation.

\subsection{3 (www.r-project.org) to conduct neighborhood analyses of focal seed set and survival. We} assumed binomial errors for the analyses of plant survival and Poisson errors for analyses of seed set. The mixed models described interactions among plants by including neighborhood indices as explanatory variables at two spatial scales in separate models. Neighborhood indices are spatial density effects of surrounding neighborhood plants that affect focal seed set and survival. For each plant, we used the Euclidian distance between the focal plant and the neighboring plants to compute response effects of intra- (same genotype) or inter-genotypic (different genotype) neighbors in a given radius around focal plants (Nottebrock et al. 2017). Moreover, we used a neighborhood index that accounts for the decline of neighbor effects with 
distance from the focal plant (Uriarte et al. 2010) and summed the amount of biomass from all individuals in a radius of $80 \mathrm{~cm}$ or $200 \mathrm{~cm}$ respectively by a Gaussian interaction kernel

171 (Lachmuth et al. 2018, Nottebrock et al. 2017, Damgaard 2004). We used random effects of

172 block and subblock to correct for environmental variation between local heterogeneous

173 conditions. Importantly, we correct for between MA line effects by including MA lines as a

174 random effect. In addition, including a random slope of biomass on each random intercept

175 corrects for the intraspecifc phenotypic variation depending on local conditions of plant focal

176 individuals. Moreover, the weighted neighborhood density by plant biomass accounts for

177 environmental variation between neighboring plants. Our model parameters and a detailed

178 model description of subblock and block models can be found in S1 supplementary text

179 (Supplementary Material).

182 the effect of intra- and inter-genotypic neighbors on survival and focal seed set with spatial

183 interaction kernels of neighborhood (plant biomass) density. By incorporating different

184 genotypes and phenotypic variation, we can quantify how important genetic variation is for

185 neighborhood models and if the phenotypic variation explains spatial interactions between

186 individuals. We assume the consequences of genetic differences to be larger between MA lines

187 than between any MA line with the founder. This is a valid assumption since each MA line

188 differs from the other by approximately $20+20=40$ mutations, while any two replicates with in

189 a MA line will differ by one generation, $\leq 2$ mutations. Thus, we simulated line effects from 
parameters derived from MA lines as random effects with the R package 'merTools' and the function 'plotREsim' in R 2018.

including plant biomass as a trait (Goldberg \& Fleetwood, 1987; Goldberg \& Landa, 1991; Cahill et al., 2005). We fitted eco-evolutionary trait-based neighborhood models at two different spatial scales for response variables (survival and seed set) for each of the two and both years. To address our objectives, we first analyzed models with differential effect in which intragenotypic neighbors (within all MA lines and founder) had a different effect on survival ( $A 1, A 2$, Table 1) and seed set (B1, B2, Table1) than inter-genotypic neighbors at small-scale (s) and large-scale (I). In addition, we analyzed models with neutral effects on survival (A1, A2, Table 1) and seed set (B1, B2, Table 1) that included total neighbor density without the split between intra-genotypic and inter-genotypic neighbors at small-scale (s) and large-scale (I). To this end, all models were fitted with two separate neighborhood indices that were calculated from intraand inter-genotypic neighbors. To justify the inclusion of individual plant biomass as trait-values for interacting plants in the model, we used AICc to compare the models with and without the trait-proxy (Burnham and Anderson 2002). We found that all models perform better including biomass as a trait-proxy $(\triangle \mathrm{AICC}>2)$. All eco-evolutionary trait-based neighborhood models contained random effects of subblock nested in block at block scale and subblock scale on the intercept, MA line identity on the intercept and the focal trait-value (plant biomass) on the slope. Additionally, because direct environmental variables were not measured during the field experiments, we included in each model the individual's biomass to correct for environmental 
212 conditions for spatial autocorrelation. All variables are scaled and centered to assure

213 comparability between predictor variables. Models of differential and neutral effects for 2004

214 and 2005 (Table 1, A1-A2, B1-B2) are fitted at small-scale ( $80 \mathrm{~cm}$ scale) and at large-scale

215 (200 cm scale). Hereafter, the $80 \mathrm{~cm}$ scale models are referred to as "small-scale" models and

216 the $200 \mathrm{~cm}$ scale models are referred to as "large scale" models. Neighborhood indices, intra-

217 and intergenotypic variation and total variation of biomass density are included as inverse

218 density variables (1/1+density). We compared models of differential and neutral effects

219 through likelihood ratio tests (LRTs). 
221 Results

222 Focal plants surrounded by plants of the same genotypes varied from 0 to 6 individuals at small-

223 scale (sub-block) and 5 to 6 individuals at large-scale (block). After determining that eco-

224 evolutionary trait-based models perform better when including plant biomass as a trait proxy to

225 calculate trait-based neighborhood indices we found that all models including plant biomass

226 perform better than models including only neighborhood density $(\triangle A I C>2$, Supplementary

227 Material S2).

228

Weighted neighbor effects of biomass density on genotypes for plant survival

230 At small-scale, survival rate is larger when surrounded by inter-genotypic than intra-genotypic

231 neighbors for both years 2004 and 2005 (model A1 and A2; Table 2 and Fig. 2a). This difference

232 of survival rate between intra- and inter-genotypic plants indicates that inter-genotypic

233 neighbors select for genotypic diversity and show stronger competitive effects on the survival

234 of intra-genotypic neighbors at small-scale. This finding is demonstrated by the superior

235 performance of the differential model relative to the neutral model (year 2004: LRT: $\chi_{1 \mathrm{DF}}^{2}=$

236 5.21, $p<0.05$; year 2005: LRT: $\left.\chi_{1 D F}^{2}=14.73, p<0.001\right)$. The differential and neutral models of

237 survival at large-scale have only non-significant effects (Table 2). In addition, comparing the AIC

238 we found that models at large-scale perform worse than at small-scale for both 2004 and 2005

239 (Table 2). We therefore will only discuss small-scale effects on survival. The distance kernel

240 (alpha) at small-scale shows that neighboring plants of different genotypes reduce plant

241 survival by $50 \%$ at $53 \mathrm{~cm}$ in 2004 and $48 \mathrm{~cm}$ in 2005. 
243 At both small and large scales, the differential model performs better than the neutral model:

244 at small scales (year 2004: LRT: $\chi_{1 D F}^{2}=52.48, p<0.001 ;$ year 2005: LRT: $\chi_{1 \text { DF }}^{2}=17.37, p<0.001$;

245 respectively) and at large scale (year 2004; LRT: $\chi_{1 D F}^{2}=679.44, p<0.001 ;$ year 2005: LRT: $\chi^{2}{ }_{1 D F}=$

$24621.17, \mathrm{p}<0.001 ;$ respectively). The small-scale model of seed set that included weighted

247 biomass densities demonstrates the reduction of seed set is larger when surrounded by inter-

248 genotypic than intra-genotypic neighbors for both years 2004 and 2005 (model B1 and B2;

249 Table 2 and Fig. 2a). The difference between intra- and inter-genotypic plants indicates that

250 inter-genotypic neighbors have a stronger competitive effect on seed set than intra-genotypic

251 neighbors at small-scale. In contrast, at large-scale seed set increases when surrounded by

252 inter-genotypic neighbors but decreases when surrounded by intra-genotypic neighbors for

253 both years 2004 and 2005 (model B1 and B2; Table 2 and Fig. 2b). Comparing the AIC between

254 small and large-scales, models at large-scale for 2004 and 2005 perform better (Table 2).

257 The distance kernel at small-scale in 2004 indicates a reduction of seed set (competition)

258 whereas at large-scale are consistent with an increase of seed set (facilitation). Neighboring

259 plants from all genotypes of $A$. thaliana at small-scale reduce $50 \%$ of focal seed set at $23 \mathrm{~cm}$

260 and at large-scale facilitate $50 \%$ of focal seed set at $86 \mathrm{~cm}$. The estimation of genotype effects

261 (MA lines and founder) simulated as conditional means and expressed as odds ratios show

262 hierarchical orders of MA line and founder competitive effects at small-scale (Fig. 3). We found 
263 no significance of simulated competitive effects of all MA lines and founder calculated from

264 survival and seed set models at small-scale (ANOVA, $p>0.1$, Fig. 4a). In addition, we found no

265 significant correlation between competitive effects of MA lines and founder at large-scale. In

266 addition, we did not find selective effects of MA lines and founder on the survival at small-scale

267 (ANOVA, $\mathrm{p}>0.1$, Fig. 4b). However, we found strong correlation between competitive effects

268 of MA lines and founder at small-scale and large-scale indicating a genetic trade-off (ANOVA,

$269 \quad F=460.07, P<0.0001$, Fig. 4c). 
272 We are able to quantify individual spatial interactions between and within genotypes (MA lines

273 and founder) using a mechanistic eco-evolutionary trait-based neighborhood model. We

274 demonstrate intraspecific genetic variation due to spontaneous mutations can shape

275 competitive abilities of genetically different individuals of $A$. thaliana. Notably, these

276 differences arose in just 25 generations of mutation and in the absence of natural selection.

277 Many of the mutations differentiating the MA lines also appear in nature, contributing to $A$.

278 thaliana genetic polymorphism (Weng et al., 2019). Plant survival is higher when surrounded by

279 inter-genotypic neighbors supporting genetic diversity. Effects on focal seed sets were reversed

280 between small and large scales; inter-genotypic neighbors have stronger negative (competitive)

281 or positive (facilitative) effects than intra-genotypic neighbors on focal seed sets at small-scale

282 or large-scale, respectively. Moreover, at small-scale, competitive effects of different MA lines

283 have similar impacts on survival and seed set. At large-scale, the intra-genotypic effect on

284 survival turns into less competitive effects on seed set. In contrast, different genotypes show

285 stronger facilitative effects within the population at large-scale, maybe because plants are more

286 competitive against other species. Below we discuss how these scale effects provide insight on

287 community assembly and potential coexistence.

Because of stronger statistical signals of effect sizes between genotypes than within genotypes,

291 niche variation may result in niche expansion or reduction (Ehlers et al. 2016). However, these 
competitive effects only occur between plants that survive to reproduction and affect seed-set.

293 The neighbor effect on plant survival suggests kin recognition despite our randomized field

294 experiment that likely reduced the spatial autocorrelation of genotypes (Hamilton 1964,

295 Kubisch et al. 2013). Seed-dispersal is limited in space because the $A$. thaliana fruit do not

296 dehiscently explode as they do in related species (Hofhuis and Hay 2017), although a seed bank

297 ensures some dispersal through time (Falahati-Anbaren et al. 2014). The neighbor effect

298 increasing genetic diversity of $A$. thaliana populations can have a positive effect on the

299 coexistence of competing species (Vellend 2006). If we assume that the increase of genetic

300 diversity due to spontaneous mutations of $A$. thaliana affects the extent of individual plant

301 niches, at large-scale the inclusion of other plant species should result in stronger competitive

302 effects of $A$. thaliana with other plant species (Hausch et al. 2018). We demonstrate that $A$.

303 thaliana individuals increase their competitive abilities with an increase of genetic diversity,

304 perhaps due to the expansion of their individual niches due to more variation in plant traits.

307 genetic trade-offs when mutations are advantageous or deleterious, which over many

308 generations could provide a strong stabilizing force maintaining both species and genetic

309 diversity in this system and promote coexistence (Lankau 2008). Thus, spontaneous mutation

310 could also provide an additional evolutionarily stabilizing effect on community dynamics. Our

311 study demonstrates the potential for a fundamental evolutionary process, mutation, to have

312 profound consequences for community structure. When $A$. thaliana is rare, selection would

313 favor genotypes that compete well and enhance the population's survival relative to that of its 
314 interspecific competitor (Lankau 2009). In contrast, when A. thaliana is common and the

315 interspecific competitor is rare, selection would favor $A$. thaliana genotypes that are good

316 intraspecific competitors. This trade-off may result in a decrease in the interspecific competitive

317 ability of $A$. thaliana, effectively increasing the competitor's fitness relative to that of $A$.

318 thaliana (Lankau 2009, Lankau and Strauss 2007). However, the genetic trade-off between

319 intra-and interspecific competitive abilities due to mutation remains unknown because our

dataset only includes performance of $A$. thaliana individuals without quantifying the

321 surrounding intraspecific environment (Chesson 2000, Adler et al. 2007).

Competitive Hierarchies of MA lines and Founder lines

324 Our result show scale dependent competition and facilitation (Nottebrock et al. 2017b)

325 providing further evidence that in a relative short evolutionary time scale spontaneous

326 mutations may change the competitive hierarchies between founders and specific MA lines due

327 to advantageous or deleterious mutations (Rutter et al. 2010). In contrast to Masclaux et al.

3282010 , we found differential responses to similar genotypes vs different genotypes in A. thaliana

329 depending on the neighbor effect on the plant survival or seed set. However, the interaction

330 between similar genotypes and different genotypes seems to depend on the strength of the

331 competitive abilities of the accessions (this study). Comparisons between MA lines and founder

332 demonstrate that competitive hierarchies follow different orders and competitive effects occur

333 at different life-history stages at different spatial scales. Eco-evolutionary processes might

334 reflect spatial selection for diversified genotypes because of niche evolution and individuals of

335 different genotypes that survived to reproduction have stronger competitive abilities (Ehlers et 
al. 2016). Moreover, the hierarchical order of competitive effects between MA lines and founders shows strong variation.

Populations can adapt evolutionarily to their environment on a time scale equivalent to that of ecological processes and affect present day species interactions, i.e., coexistence (Fussmann et al. 2007). Yet, sustained and rapid climate change could deplete genetic variance faster than it can be replenished by mutation (Fournier-Level et al. 2016). In our study, we show that only 25 generations are enough to influence present day plant interactions of $A$. thaliana in the presence of other plant species. Possible effects of mutations on trait mechanisms might reflect the increased seed set of $A$. thaliana at large-scale, because of the presence of another plant species allowing the establishment and growth of $A$. thaliana (Ehlers et al. 2016). Many traits can influence competition and facilitation between individuals of different genotypes (e.g. competitors (Lankau and Strauss 2007, Van Dam and Baldwin 1998; Tiffin 2002, Strauss and Irwin 2004). Such spatial mechanisms might be described by the scale difference of competition at small-scale and facilitation at large-scale between different $A$. thaliana genotypes.

354 Competition and facilitation are therefore dependent on the extension or reduction of 355 individual niches, which is based on the ability to advance or distract traits due to spontaneous 356 mutations. However, we are not able to directly link our results to benefactor or antagonistic plants, because we focused in our experiment on $A$. thaliana densities and fitness components. 
358 Nevertheless, genotypic-specific interactions are well studied. A number of studies of $A$.

thaliana MA lines demonstrate mutation effects on plant traits such as leaf weight, flowering

time, trichome density, number of leaves at bolting, duration of vegetative and flowering

period (Camara and Pigliucci 1999, Rutter et al. 2010, Stearns and Fenster 2016). It is

conceivable that the increase in variance of these traits through spontaneous mutations could

contribute to the evolution of competitive hierarchies. heterogeneous environment with various genotypes (Hamilton 1964). Especially, at large-scale, we show that different genotypes facilitate seed set that stabilize the population dynamics by increasing the performance of neighboring plant individuals (Latzel et al. 2013, Castellanos et al.

373 2014). Thus, selection for diversified genotypes might reveal a coexistence mechanism, because 374 mutations at a short time scale ( 25 generations) are able to alter the competitive hierarchies of 375 A. thaliana individuals of different genotypes. These genotypes might expand or reduce their niches and can therefore act more competitive against strangers (Ehlers et al 2016). Moreover,

377 variation in competitive ability among genotypes due to mutation can lead to intransitive competitive hierarchies at a small-scale, and allow coexistence of competitors at large-scale 
1990, Laure et al. 2017). This might also reflect the genetic trade-off between competitive

small-scale results in weaker competition between different species at large-scale. Thus, the

constant input of mutation to genetic variation of competitive hierarchies suggests that genetic

variation is likely never a limiting factor in the evolution of those traits that influence plant community dynamics.

In ecology, two primary contrasting models are crucial to population and community dynamics 
401 change hierarchies of intra-specific interactions in natural plant populations over a short

402 evolutionary time scale. Our results are concordant with the niche theory stressing the

403 changing of competitive hierarchies among phenotypes in a given environment (Levine and

404 HilleRisLambers 2009, Nottebrock et al. 2017). Moreover, selection and trait evolution favor

405 population survival of $A$. thaliana in the wild, which stresses the importance of combining

406 spatial ecological and evolutionary mechanisms for our understanding of population and

407 community dynamics.

408 
Conclusion

Rapid evolution of $A$. thaliana due to spontaneous mutation alone has profound consequences for population and community dynamics. Competition of $A$. thaliana individuals is genotypedependent and $A$. thaliana is not a single weak competitor among co-occurring plant species (Ehlers et al. 2016, Soliveres et al 2017). Moreover, the discovery of the underlying ecoevolutionary nature of competition in A. thaliana supports a shift from species-based to individual-based community ecology. This would lead to a more predictive ecological theory (Violle et al 2017). We show that including the intraspecific genetic variation and phenotypic variation as separate aspects to a trait-based neighborhood model increases the predictive power to understand population and community dynamics. In particular, non-neutral intraspecific processes may determine species coexistence, because genetic diversity is promoted by having stronger competitive abilities at small-scale and stabilizing population survival at large-scale (Clark et al. 2010). Additionally, our study demonstrates higher genetic diversity increases population survival due to rapid evolution with implications to forecast the fate of species and functional diversity in response to environmental changes (Violle et al 2012). Depending on the time of environmental changes, species may adapt to environmental change by shifting their fundamental niches (Clark 2010). The combination of intraspecific genotypic variation and spatial interactions might advance our understanding of community dynamics, especially of rapid evolution (Koch et al. 2014, Turcotte and Levine 2016). Incorporating genetic variation and the eco-evolutionary process for determining standing levels of genetic variation will provide a better understanding of species interactions underlying the maintenance of biodiversity. 
bioRxiv preprint doi: https://doi.org/10.1101/497610; this version posted December 20, 2018. The copyright holder for this preprint (which was not certified by peer review) is the author/funder, who has granted bioRxiv a license to display the preprint in perpetuity. It is made available under aCC-BY-ND 4.0 International license.

432 Keywords (10 for indexing purpose): trait-based community ecology, spontaneous mutations,

433 competition, facilitation, niche evolution, intraspecific genetic variation, intraspecific

434 phenotypic variation, Arabidopsis thaliana, mutation accumulation lines, eco-evolutionary

435 dynamics.

436 
438

439

440

441

442

443

444

445

446

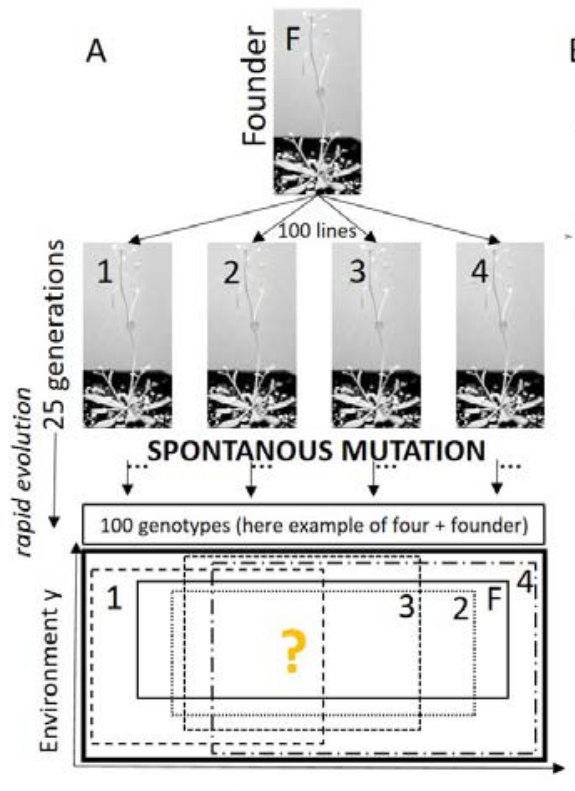

Environment $\mathrm{x}$

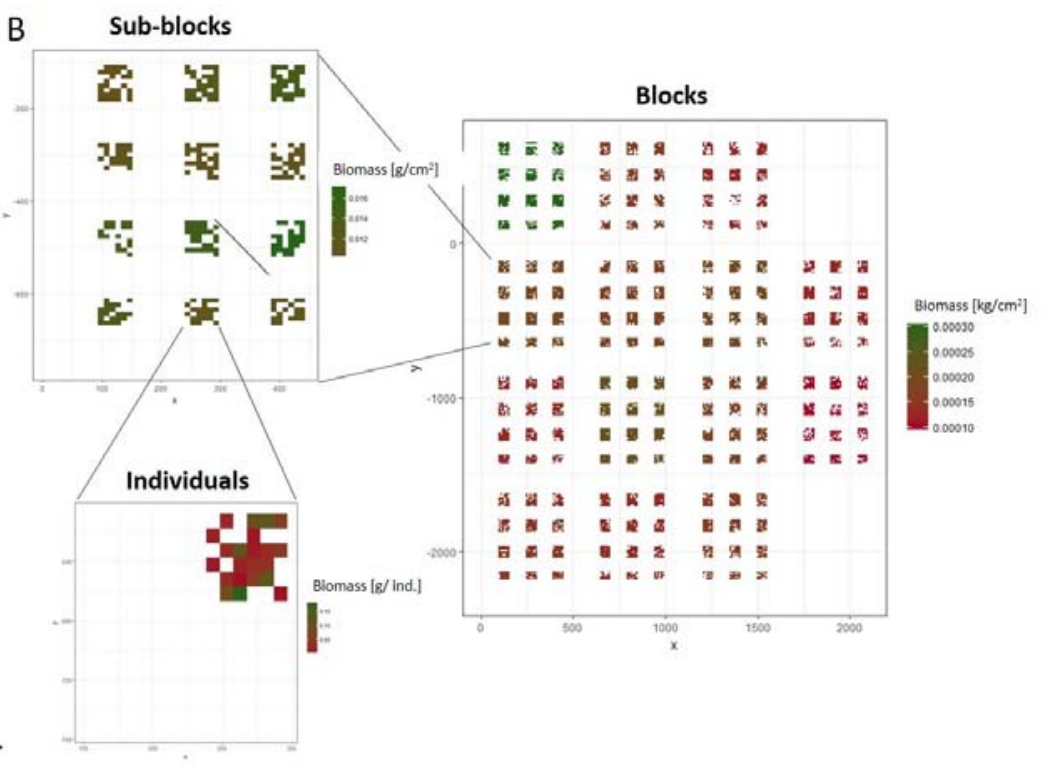

Figure 1. (A) 100 Mutation accumulation lines (here example of four MA-lines) of Arabidopsis

thaliana derived from a single founder (Columbia) after 25 generations with a mutation rate of

0.7 per generation (Weng et al. 2019). Each MA line has a unique genotype that differs from

the founder. Fundamental niche of individual genotypes (different dashed and dotted lines) and

founder genotype (black line) are distributed within the hypothetical fundamental niche of a

population of $A$. thaliana (bold black line). Axes represent niche axis (resource-use,

environmental factors, etc.). (B) Planting design of $A$. thaliana in a natural field experiment

showing spatial biomass variation of individual survivors in 2004. 


\section{Year $2004 \quad$ Year $2005 \quad$ Model}

\begin{tabular}{llll}
\hline Individual & $\mathrm{A} 1(\mathrm{~s}, \mathrm{I})$ & $\mathrm{A} 2(\mathrm{~s}, \mathrm{I})$ & Differential \\
\cline { 2 - 4 } survival & $\mathrm{A} 1(\mathrm{~s}, \mathrm{I})$ & $\mathrm{A} 2(\mathrm{~s}, \mathrm{I})$ & Neutral \\
& $\mathrm{B} 1(\mathrm{~s}, \mathrm{I})$ & $\mathrm{B} 2(\mathrm{~s}, \mathrm{I})$ & Differential \\
\hline Seed set & $\mathrm{B} 1(\mathrm{~s}, \mathrm{I})$ & $\mathrm{B} 2(\mathrm{~s}, \mathrm{I})$ & Neutral
\end{tabular}

449 Table 1. Table of all different eco-evolutionary trait-based neighborhood models including

450 intraspecific variation to test our three objectives of density effects on survival and seed set of

451 A. thaliana. Differential models split the neighbor responses into within genotypes and

452 between genotypes. Neutral models describe the overall neighbor response without

453 differentiating between genotypes. All models include random effects of plant biomass on the

454 slope, subblock or block and MA-line on the intercept (see Fig. 1 for design), and respectively

455 depending on small (s) or large-scale (I) (for more details see method section). 
Survival model

(A1) 2004

(s) S
Intra
$-0.02^{\mathrm{ns}}$

(s) Small scale $(80 \mathrm{~cm})$

AIC

(I) Larger scale $(200 \mathrm{~cm}) \quad$ AIC

Weighted density

\begin{tabular}{l|l|l}
\hline MA & neutral & $-0.02^{\text {ns }}$
\end{tabular}

Inter

Intra

Inter

Lines

\begin{tabular}{|c|c|c|c|c|c|c|}
\hline \multirow[t]{2}{*}{ (A2) 2005} & \multicolumn{2}{|c|}{ (s) Small scale $(80 \mathrm{~cm})$} & \multirow[t]{2}{*}{ AIC } & \multicolumn{2}{|c|}{ (I) Larger scale $(200 \mathrm{~cm})$} & \multirow[t]{2}{*}{ AIC } \\
\hline & Intra & Inter & & Intra & Inter & \\
\hline
\end{tabular}

Weighted density

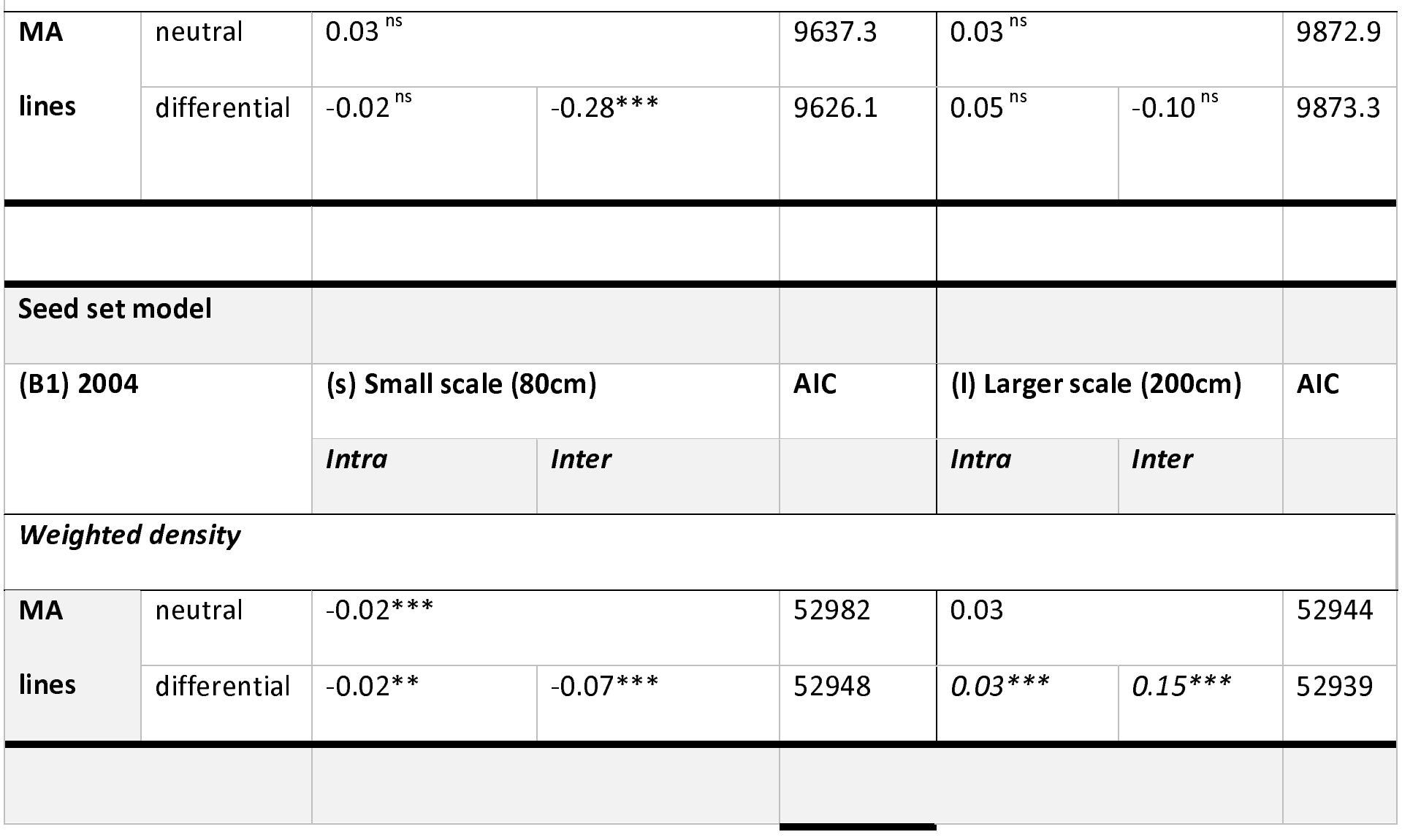




\begin{tabular}{|c|c|c|c|c|c|c|}
\hline \multirow[t]{2}{*}{ (B2) 2005} & \multicolumn{2}{|c|}{ (s) Small scale $(80 \mathrm{~cm})$} & \multirow[t]{2}{*}{ AIC } & \multicolumn{2}{|c|}{ (I) Larger scale $(200 \mathrm{~cm})$} & \multirow[t]{2}{*}{ AIC } \\
\hline & Intra & Inter & & Intra & Inter & \\
\hline
\end{tabular}

\section{Weighted density}

\begin{tabular}{|c|c|c|c|c|c|c|c|}
\hline MA & neutral & $-0.008^{*}$ & & 49103 & $-0.008^{*}$ & & 49049 \\
\hline lines & differential & $-0.009 * *$ & $-0.09 * * *$ & 49095 & $-0.01^{*}$ & $0.16 * * *$ & 49026 \\
\hline
\end{tabular}

456

457 Table 2. The coefficient and AIC from eco-evolutionary trait-based neighborhood models of the

458 Arabidopsis thaliana field experiment in the years 2004 and 2005. Models include intraspecific

459 genetic variation defined as intra-genotyptic (within genotypes) and inter-genotypic (between

460 genotypes) variation. Models are shown at two different spatial scales: weighted subblock

461 density ( $80 \mathrm{~cm}$ radius) and weighted block density $(200 \mathrm{~cm}$ radius). All scales include variables of

462 neutral effects (absence of intra- and inter-genotypic variation (total sum of all neighboring

463 genotypes)) and differential effects (presence of intra- and inter-genotypic variation (split of

464 neighboring genotypes in intra- and inter-genotypic genotypes). Thus, neighbor identity is split

465 into intra- and inter-genotypes as differential effects or are combined as neutral effects, which

466 includes all neighbors. P-value levels $<0.05^{*},<0.005^{* *},<0.001^{* * *}$ and $>0.05^{\mathrm{ns}}$. 


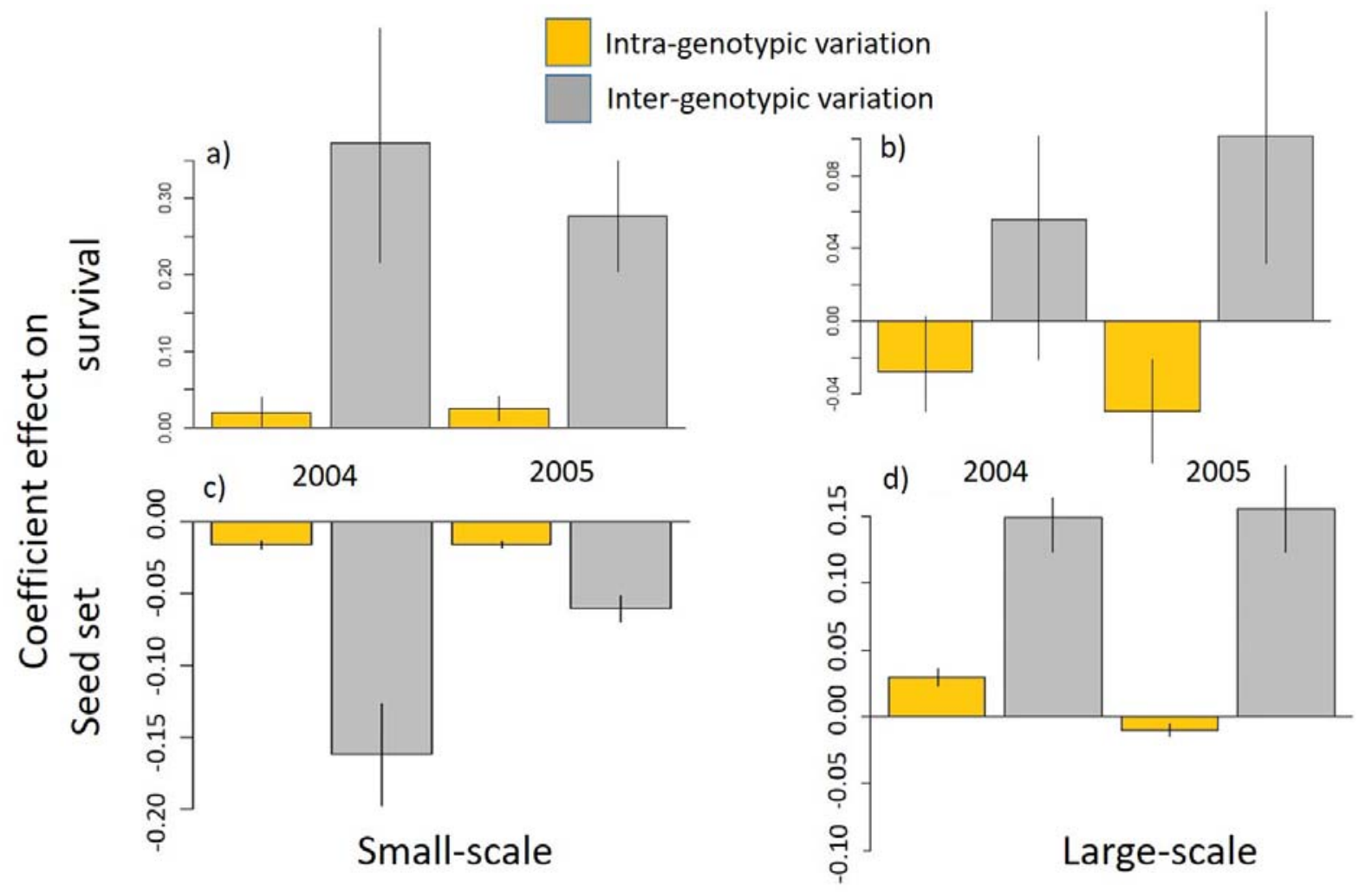

468 Figure 2. The barplots show standardized coefficient effect sizes and associated standard errors

469 of eco-evolutionary trait-based neighborhood models at small-scale $(a, c)$ and large-scale $(b, d)$

470 for all MA-lines for the survival model (a, b) and the seed set model (c, d). Intra-genotypic

471 effects (yellow) and inter-genotypic effects (grey) are presented for 2004 and 2005. Coefficient

472 effect sizes indicate reduction or an increase of seed set depending on biomass density of intra-

473 or inter-genotypic neighbors. In addition, coefficient effect sizes indicate a reduction of survival

474 of intra or inter-genotypic neighbors. 
MA line effect sizes of seed set (a), (b) and survival (c) model
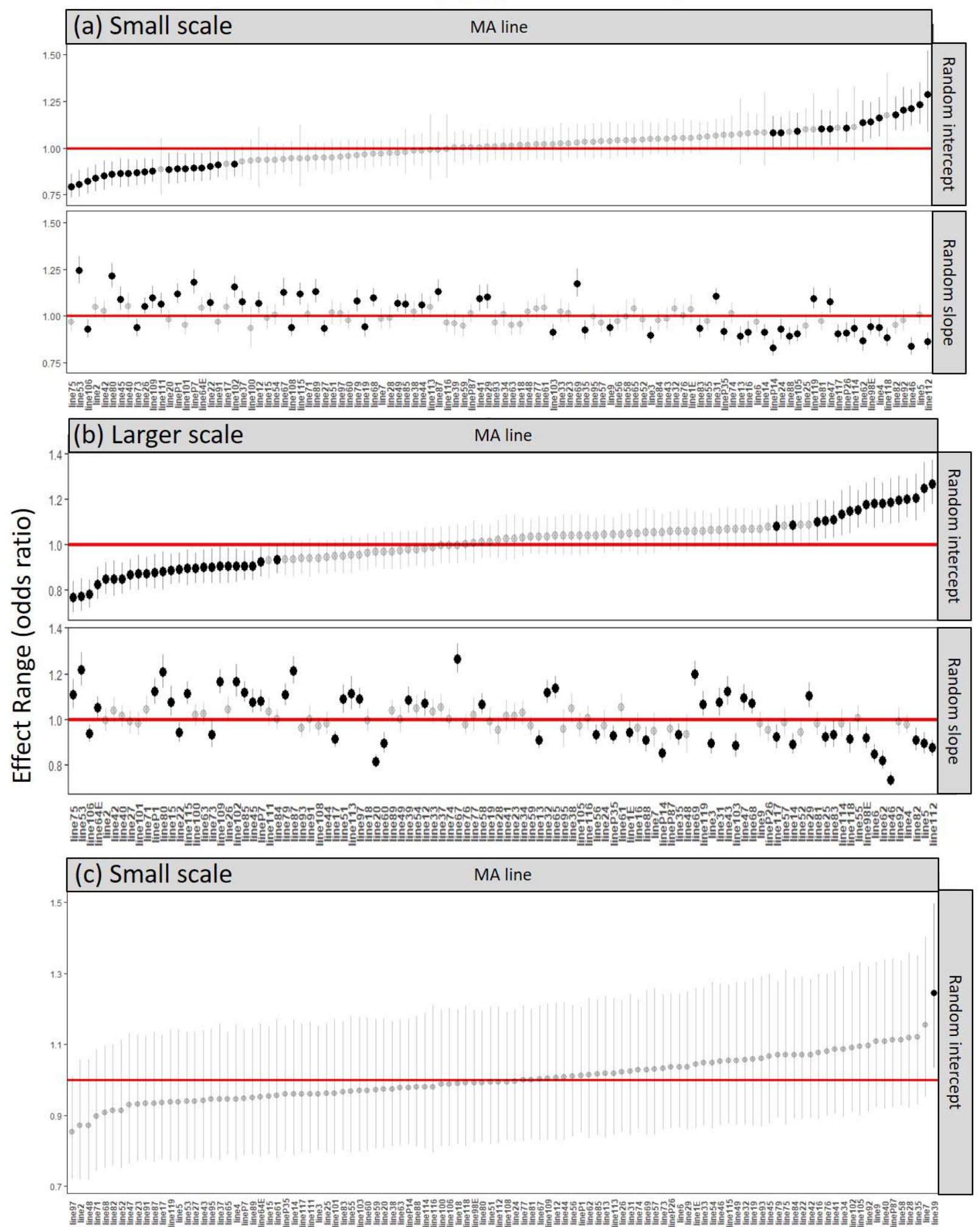
477 Figure 3. The effect range on seed set (a), (b) and survival (c) computed from eco-evolutionary

478 trait-based neighborhood models shown as odds ratios for year 2004 (for 2005 see

479 Supplementary Material). (a) Shows the hierarchical competitive order of MA lines and founder

480 for the seed set model including range effects of each MA line and founder as random

481 intercepts and the trait proxy biomass as random slopes at small-scale and (b) at large-scale. (c)

482 Shows the hierarchical selective order of MA lines and founder for the survival model including

483 range effects of each MA line and founder as random intercepts at small-scale. 
Correlation between MA line effect sizes of significant seed set and survival model

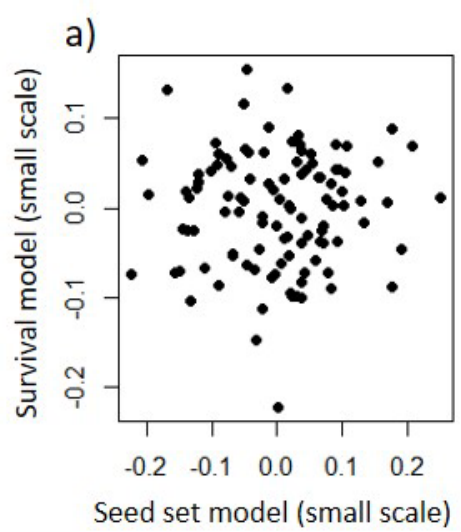

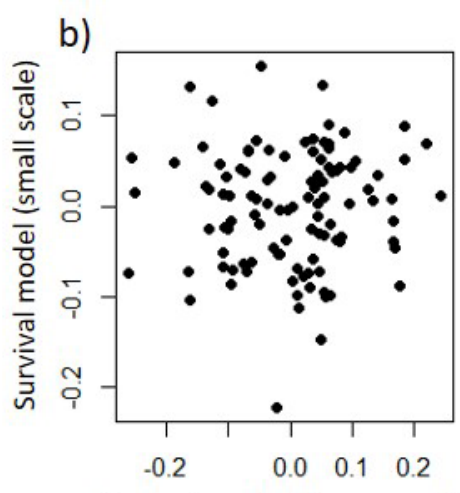

Seed set model (larger scale)

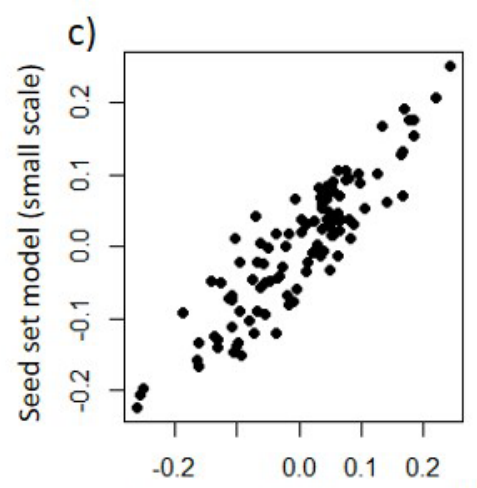

Seed set model (larger scale)

Figure 4. Correlation of the simulated conditional mean represented as odds ratio of same

\section{7}

the effect sizes on the survival or seed set. a) Relationship between survival and seed set

model. b) Relationship between survival and seed set model. c) Relationship between small-

scale survival and large-scale seed set model. Since we are plotting effect sizes, the positive

correlation in panel $\mathrm{c}$ reflects a trade-off between competition and facilitation (see results). 
497 Adler P., B., HilleRisLambers Janneke \& Levine J. M. (2007). A niche for neutrality. Ecology Letters, $498 \quad 10,95-104$.

499 Agrawal, A.A., Hastings, A.P., Johnson, M.T.J., Maron, J.L. \& Salminen, J.-P. (2012). Insect

500 herbivores drive real-time ecological and evolutionary change in plant populations. Science, $501338,113-116$. Garden, 63, 224-247. Bailey JKet al.2009From genes to ecosystems: a synthesis of the effects of plant genetic factors across levels of organization. Phil. Trans. R. Soc. B 364, 1607-1616.

506 (doi:10.1098/rstb.2008.0336)

507 Baron E., Richirt J., Villoutreix R., Amsellem L., Roux F. \& Bennett A. (2015). The genetics of intraand interspecific competitive response and effect in a local population of an annual plant species. Functional Ecology, 29, 1361-1370. Bataillon, T. (2003). Shaking the 'deleterious mutations' dogma? Trends in Ecology \& Evolution, $18,315-317$.

512 Bates, D., Mächler, M., Bolker, B. \& Walker, S. (2014). Fitting Linear Mixed-Effects Models using $513 \quad$ Ime4. arXiv:1406.5823 [stat].

514 Bellard C., Bertelsmeier C., Leadley P., Thuiller W.\& Courchamp F. (2012). Impacts of climate 515 change on the future of biodiversity. Ecology Letters, 15, 365-377. 
516 Bolnick, D.I., Amarasekare, P., Araújo, M.S., Bürger, R., Levine, J.M., Novak, M., et al. (2011). Why

517 intraspecific trait variation matters in community ecology. Trends in Ecology \& Evolution, 26,

$518 \quad$ 183-192.

519 Bossdorf Ol., Prati D., Auge H. \& Schmid B. (2004). Reduced competitive ability in an invasive 520 plant. Ecology Letters, 7, 346-353.

521 Burnham and Anderson (2002). Model Selection and Multimodel Inference: A Practical

522 Information-Theoretical Approach. 2d ed. New York: Springer-Verlag

523 Bruno, J.F., Stachowicz, J.J. \& Bertness, M.D. (2003). Inclusion of facilitation into ecological

524 theory, 7.

525 Cahill, F. J., Kembel, S. W. and Gustafson, D. J. (2005), Differential genetic influences on

526 competitive effect and response in Arabidopsis thaliana. Journal of Ecology, 93: 958-967.

527 doi:10.1111/j.1365-2745.2005.01013.x

528 Cahill, J.F., Elle, E., Smith, G.R. \& Shore, B.H. (2008). Disruption of a belowground mutualism

529 alters interactions between plants and their floral visitors. Ecology, 89, 1791-1801.

530 Camara, M. d. and M. Pigliucci. (1999). Mutational contributions to genetic variance-covariance

531 matrices: An experimental approach using induced mutations in Arabidopsis thaliana.

$532 \quad$ Evolution $53(6), 1692-1703$

533 Caradus, J. \& Woodfield, D. Plant and Soil (1998) 200: 63. 
535 Carr, D. E. and Dudash, M. R. (1996), Inbreeding depression in two species of Mimulus

536 (Scrophulariaceae) with contrasting mating systems. American Journal of Botany, 83: 586-

537 593. doi:10.1002/j.1537-2197.1996.tb12743.x beneficiary plant population? Journal of Ecology, 102, 1214-1221.

541 Chapin, F. S. III, Autumn, K., \& Pugnaire, F. (1993). Evolution of suites of traits in response to environmental stress. American Naturalist, 142, S78-S92.

543 Chase, J. M. et al. 2002. The interaction between predation and competition: a review and 544 synthesis. - Ecol. Lett. 5: 302-315.

545 Chave, J., Muller-Landau, H.C. \& Levin, S.A. (2002). Comparing Classical Community Models:

546 Theoretical Consequences for Patterns of Diversity. The American Naturalist, 159, 1-23.

547 Chesson, P. (2000). Mechanisms of Maintenance of Species Diversity. Annual Review of Ecology $548 \quad$ and Systematics, 31, 343-366.

549 Clark, J.S. (2010). Individuals and the Variation Needed for High Species Diversity in Forest Trees. 550 Science, 327, 1129-1132.

551 Colwell, R.K. \& Rangel, T.F. (2009). Hutchinson's duality: The once and future niche. PNAS, 106, 19651-19658.

553 Connell, J. (1980). Diversity and the Coevolution of Competitors, or the Ghost of Competition 
555 Damgaard, C. (2004). Inference from plant competition experiments: the effect of spatial

556 covariance. Oikos, 107, 225-230.

557 Dudley, S. A., Murphy, G. P., File, A. L. and Robinson, D. (2013), Kin recognition and competition

558 in plants. Funct Ecol, 27: 898-906. doi:10.1111/1365-2435.12121

559 Ehlers, B.K., Damgaard, C.F. \& Laroche, F. (2016). Intraspecific genetic variation and species

560 coexistence in plant communities. Biology Letters, 12, 20150853.

561 Exposito-Alonso M, Becker C, Schuenemann VJ, Reiter E, Setzer C, Slovak R, et al. (2018) The rate

562 and potential relevance of new mutations in a colonizing plant lineage. PLoS Genet 14(2):

563 e1007155. https://doi.org/10.1371/journal.pgen.1007155

564 Falahati-Anbaran, M., Lundemo, S. and Stenøien, H. K. (2014), Seed dispersal in time can

565 counteract the effect of gene flow between natural populations of Arabidopsis thaliana. New

566 Phytol, 202: 1043-1054. doi:10.1111/nph.12702

567 Fisher C.K. and Mehta P.(2014). The transition between the niche and neutral regimes in ecology.

$568 \quad$ Proc Natl Acad Sci USA 111:13111-13116

569 Fournier-Level A, et al.(2016) Predicting the evolutionary dynamics of seasonal adaptation to

570 novel climates in Arabidopsis thaliana. Proc Natl Acad Sci USA 113(20):E2812-E2821.

571 Fridley, J.D. \& Grime, J.P. (2010). Community and ecosystem effects of intraspecific genetic

572 diversity in grassland microcosms of varying species diversity. Ecology, 91, 2272-2283. 
573 Fussmann G. F., Loreau M. \& Abrams P. A. (2007). Eco-evolutionary dynamics of communities and 574 ecosystems. Functional Ecology, 21, 465-477.

575 Genung, M. A., Schweitzer, J. A., Úbeda, F. , Fitzpatrick, B. M., Pregitzer, C. C., Felker-Quinn, E. 576 and Bailey, J. K. (2011), Genetic variation and community change - selection, evolution, and 577 feedbacks. Functional Ecology, 25: 408-419. doi:10.1111/j.1365-2435.2010.01797.x

578 Hamilton, W. D. 1964. The genetical evolution of social behaviour. I \& II. J. Theor. Biol. 7:1-52.

579 Hart, S. P., Schreiber, S. J., Levine, J. M. and Coulson, T. (2016), How variation between individuals 580 affects species coexistence. Ecol Lett, 19: 825-838. doi:10.1111/ele.12618

581 Hart, S. P., Freckleton, R. P., Levine, J.M. \& Kroon, H. (2018). How to quantify competitive ability. 582 Journal of Ecology, 0.

583 Hausmann N. J., Juenger T. E., Sen S., Stowe K. A., Dawson T. E., et al., 2005. Quantitative trait loci 584 affecting $\delta C^{13}$ and response to differential water availability in Arabidopsis thaliana. Evolution $585 \quad 59: 81-96$

586 Hofhuis, H. and Hay, A. (2017), Explosive seed dispersal. New Phytol, 216: 339-342. doi:10.1111/nph.14541

588 Holt, R.D. (2009). Bringing the Hutchinsonian niche into the 21st century: Ecological and 589 evolutionary perspectives. PNAS, 106, 19659-19665.

590 Hubbell S.P. (2005). Neutral theory in community ecology and the hypothesis of functional 591 equivalence. Functional Ecology, 19, 166-172.

592 Hutchinson G.E. (1957). Concluding remarks. Cold Spring Harbor Symp Quant Biol 22:415-427. 
593 Johnson, M.T.J. Stinchcombe, J.R. An emerging synthesis between community ecology and

594 evolutionary biology Trends Ecol. Evol., 22 (2007), pp. 250-257

595 Johnson, M.T.J., Vellend, M. \& Stinchcombe, J.R. (2009). Evolution in plant populations as a driver

596 of ecological changes in arthropod communities. Philosophical Transactions of the Royal

597 Society of London B 364:1593-1605.

598 Kerwin et al. (2015) Natural genetic variation in Arabidopsis thaliana defense metabolism genes

599 modulates field fitness | eLife Lens. https://lens.elifesciences.org/05604/. Last accessed 4

$600 \quad$ May 2018.

601 Koch, H., Frickel, J., Valiadi, M. \& Becks, L. (2014). Why rapid, adaptive evolution matters for 602 community dynamics. Front. Ecol. Evol. , 2.

603 Kubisch A. et al. (2013). Kin competition as a major driving force for invasions. Am. Nat. 181: 700-

$604 \quad 706$

605 Lachmuth S., Henrichmann C., Horn J., Pagel J., Schurr F.M. \& Rafferty N. (2018). Neighbourhood

606 effects on plant reproduction: An experimental-analytical framework and its application to

607 the invasive Senecio inaequidens. Journal of Ecology, 106, 761-773.

608 Lankau, R.A. (2011). Rapid Evolutionary Change and the Coexistence of Species. Annual Review of

609 Ecology, Evolution, and Systematics, 42, 335-354.

610 Lankau, R.A. \& Strauss, S.Y. (2007). Mutual feedbacks maintain both genetic and species diversity

611 in a plant community. Science, 317, 1561-1563. 
612 Lankau R. A., Wheeler E., Bennett A. E. \& Strauss S. Y. (2010). Plant-soil feedbacks contribute to

613 an intransitive competitive network that promotes both genetic and species diversity. Journal

614 of Ecology, 99, 176-185.

615 Latzel, V., Allan, E., Bortolini Silveira, A., Colot, V., Fischer, M. \& Bossdorf, O. (2013). Epigenetic

616 diversity increases the productivity and stability of plant populations. Nat Commun, 4, 2875.

617 Laure, G. Zimmermann N. E., Levine J. M., Adler P.r B. \& Wootton T (2017). The effects of

618 intransitive competition on coexistence. Ecology Letters, 20, 791-800.

619 Levine, J.M. \& HilleRisLambers, J. (2009). The importance of niches for the maintenance of

620 species diversity. Nature, 461, 254-257.

621 Lion, S. (2018) Theoretical approaches in evolutionary ecology: Environmental feedback as a

622 unifying perspective. Am. Nat.191, 21-44.

623 Lynch, M., and B. Walsh. (1998). Genetics and analysis of quantitative traits. Sinauer Associates,

624 Inc., Sunderland, MA.

625 Masclaux, F., Hammond, R. L., Meunier, J. , Gouhier-Darimont, C., Keller, L. and Reymond, P.

626 (2010), Competitive ability not kinship affects growth of Arabidopsis thaliana accessions. New

627 Phytologist, 185: 322-331. doi:10.1111/j.1469-8137.2009.03057.x

628 Mukai, T. (1964). The Genetic Structure of Natural Populations of Drosophila Melanogaster. I.

629 Spontaneous Mutation Rate of Polygenes Controlling Viability. Genetics, 50, 1-19.

630 Nei, M. (2013). Mutation-driven evolution. Oxford University Press 
631 Nottebrock H., Schmid B., Mayer K., Devaux C., Esler K. J., Böhning-Gaese K., et al. (2017a). Sugar

632 landscapes and pollinator-mediated interactions in plant communities. Ecography, 40, 1129-

6331138.

634 Nottebrock H., Schmid B,., Treurnicht M., Pagel J., Esler K. J., Böhning-Gaese K., et al. (2017b).

635 Coexistence of plant species in a biodiversity hotspot is stabilized by competition but not by

636 seed predation. Oikos, 126.

637 Ossowski, S., K. Schneeberger, J. I. Lucas-Lled’o, N.Warthmann, R. M. Clark, R. G. Shaw, D. Weigel,

638 and M. Lynch. (2010). The rate and molecularspectrum of spontaneous mutations in

639 Arabidopsis thaliana. Science 327:92-94.

640 Roles, A. J., and J. K. Conner. (2008). Fitness effects of mutation accumulation in a natural

641 outbred population of wild radish (Raphanus raphanistrum): comparison of field and

642 greenhouse environments.. Evolution, 62: 1066-1075. doi:10.1111/i.1558-5646.2008.00354.x

643 Roles, A. J., M. T. Rutter, C. B. Fenster and J. K. Conner. (2016). Field measurement of genotype by

644 environment interaction for fitness caused by spontaneous mutations in Arabidopsis thaliana.

$645 \quad$ Evolution, 70: 1039-1050.

646 R Development Core Team (2011). R: A language and environment for statistical computing.

647 Vienna, Austria: R Foundation for Statistical Computing.

648 Rutter, M. T., F. H. Shaw and C. B. Fenster. (2010). Spontaneous mutation parameters for

649 Arabidopsis thaliana measured in the wild. Evolution, 64: 1825-1835. 
650

651

652

653

654

655

656

657

658

659

660

661

662

663

664

665

666

667

668

669

Rutter, M., A. Roles, J. Conner, R. Shaw, F. Shaw, K. Schneeberger, S. Ossowski, D. Weigel and C.

B. Fenster. (2012). Brief Communication: Fitness of Arabidopsis thaliana mutation

accumulation lines whose spontaneous mutations are known. Evolution, 66: 2335-2339.

Rutter, M. T., A. J. Roles, and C. B. Fenster. (2018). Quantifying natural seasonal variation in mutation parameters with mutation accumulation lines. Ecology and Evolution, https://doi.org/10.1002/ece3.4085

Schaack, S., Allen, D.E., Latta, L.C., Morgan, K.K. \& Lynch, M. (2013). The effect of spontaneous mutations on competitive ability. Journal of Evolutionary Biology, 26, 451-456.

Shaw, R.G., Byers, D.L. \& Darmo, E. (2000). Spontaneous Mutational Effects on Reproductive Traits of Arabidopsis thaliana..Genetics 155:369-378.

Shaw, F. H., C. J. Geyer, and R. G. Shaw. (2002). A comprehensive model of mutations affecting fitness and inferences for Arabidopsis thaliana. Evolution 56:453-463.

Shefferson Richard P., Salguero-Gómez Roberto \& Rees Mark. (2015). Eco-evolutionary dynamics in plants: interactive processes at overlapping time-scale and their implications. Journal of Ecology, 103, 789-797.

Soliveres, S., Smit, C. \& Maestre, F.T. (2015). Moving forward on facilitation research: response to changing environments and effects on the diversity, functioning and evolution of plant communities. Biol Rev Camb Philos Soc, 90, 297-313.

68 Soliveres Set al. (2015). Intransitive competition is widespread in plant communities and maintains their species richness. Ecol. Lett. 18, 790-798. doi:10.1111/ele.12456 
670 Stearns, F. W. and C. B. Fenster. (2016). The effect of induced mutations on quantitative traits in

671 Arabidopsis thaliana: Natural versus artificial conditions. Ecology and Evolution, doi:

$672 \quad 10.1002 /$ ece3.2558

673 Strauss, S.Y. \& Irwin, R.E. (2004). Ecological and evolutionary consequences of multispecies

674 plant-animal interactions. Annu. Rev. Ecol. Evol. Syst., 35, 435-466.

675 Taylor, D.R., Aarssen, L.W. \& Loehle, C. (1990). On the Relationship between r/K Selection and

676 Environmental Carrying Capacity: A New Habitat Templet for Plant Life History Strategies.

677 Oikos, 58, 239-250.

678 Tiffin, P. (2002). Competition and time of damage affect the pattern of slection acting on plant

679 defense against herbivores. Ecology, 83: 1981-1990. doi:10.1890/0012-

680 9658(2002)083[1981:CATODA]2.0.CO;2

681 Tilman D. (2004). Niche tradeoffs, neutrality, and community structure: a stochastic theory of 682 resource competition, invasion, and community assembly. Proc. Natl Acad. Sci. USA 101, 10 854-10 861. doi:10.1073/Pnas.0403458101

684 Turcotte, M.M. \& Levine, J.M. (2016). Phenotypic Plasticity and Species Coexistence. Trends Ecol. 685 Evol. (Amst.), 31, 803-813.

686 Uriarte, M., Swenson, N.G., Chazdon, R.L., Comita, L.S., John Kress, W., Erickson, D., et al. (2010).

687 Trait similarity, shared ancestry and the structure of neighbourhood interactions in a 688 subtropical wet forest: implications for community assembly. Ecol. Lett., 13, 1503-1514. 
689 Van Dam, N. M. and Baldwin, I. T. (1998), Costs of jasmonate-induced responses in plants

690 competing for limited resources. Ecology Letters, 1: 30-33. doi:10.1046/j.1461-

691 0248.1998.00010.x

692

Vellend Mark \& Geber Monica A. (2005). Connections between species diversity and genetic diversity. Ecology Letters, 8, 767-781.

694 Violle, C., Enquist, B.J., McGill, B.J., Jiang, L., Albert, C.H., Hulshof, C., et al. (2012). The return of 695 the variance: intraspecific variability in community ecology. Trends in Ecology \& Evolution, 27, $696 \quad 244-252$.

697 Violle, C. \& Jiang, L. (2009). Towards a trait-based quantification of species niche. J Plant Ecol, 2, $698 \quad 87-93$.

699 Violle, C., Thuiller, W., Mouquet, N., Munoz, F., Kraft, N.J.B., Cadotte, M.W., et al. (2017).

700 Functional Rarity: The Ecology of Outliers. Trends in Ecology \& Evolution, 32, 356-367.

701 Weng, M.-L., Becker, C., Hildebrandt, J., Rutter,M.T., Shaw, R.G., Weigel, D. and C. B. Fenster. 702 Fine-Grained Analysis of Spontaneous Mutation Spectrum and Frequency in Arabidopsis 703 thaliana Genetics. Early online (2018); https://doi.org/10.1534/genetics.118.301721

704 Whitham, T. G., Young, W. P., Martinsen, G. D., Gehring, C. A., Schweitzer, J. A., Shuster, S. M., 705 Wimp, G. M., Fischer, D. G., Bailey, J. K., Lindroth, R. L., Woolbright, S. and Kuske, C. R. (2003), 706 Community and ecosystem genetics: A consequence of extended phenotype. Ecology, 84:

707 559-573. doi:10.1890/0012-9658(2003)084[0559:CAEGAC]2.0.CO;2 
708 Whitham, T.G., Bailey, J.K., Schweitzer, J.A., Shuster, S.M., Bangert, R.K., LeRoy, C.J. et al. (2006).

709 A framework for community and ecosystem genetics: from genes to ecosystems. Nat. Rev.

710 Genet. , 7, 510-523.

711 Whitlock R. \& Lortie C. (2014). Relationships between adaptive and neutral genetic diversity and

712 ecological structure and functioning: a meta-analysis. Journal of Ecology, 102, 857-872.

713

714

715

716 